\title{
Analysis of Cross-Correlations in Emerging Markets Using Random Matrix Theory
}

\author{
Thomas Chinwe Urama*, Patrick Oseloka Ezepue, Chimezie Peters Nnanwa \\ Statistics, Information Modelling and Financial Mathematics Research Group, Department of Engineering \& Mathematics, \\ Sheffield Hallam University, Sheffield, UK \\ Email: ^thomas.c.urama@student.shu.ac.uk, p.ezepue@shu.ac.uk, chimezie.p.nnanwa@student.shu.ac.uk
}

How to cite this paper: Urama, T.C., Ezepue, P.O. and Nnanwa, C.P. (2017) Analysis of Cross-Correlations in Emerging Markets Using Random Matrix Theory. Journal of Mathematical Finance, 7, 291307.

https://doi.org/10.4236/jmf.2017.72015

Received: December 3, 2016

Accepted: May 12, 2017

Published: May 16, 2017

Copyright $\odot 2017$ by authors and Scientific Research Publishing Inc. This work is licensed under the Creative Commons Attribution International License (CC BY 4.0).

http://creativecommons.org/licenses/by/4.0/

\section{Open Access}

\begin{abstract}
This paper investigates the universal financial dynamics in two dominant stock markets in Sub-Saharan Africa, through an in-depth analysis of the cross-correlation matrix of price returns in Nigerian Stock Market (NSM) and Johannesburg Stock Exchange (JSE), for the period 2009 to 2013. The strength of correlations between stocks is known to be higher in JSE than that of the NSM. The stock price dynamics in the NSM is important for modeling Nigerian derivatives in the future, and in the light of this, it is pertinent to note that the interactions of other stocks with the oil sector are weak, whereas the banking stocks have strong positive interactions with the other sectors in the stock exchange. For the JSE, it is the oil sector and beverages that have greater sectorial correlations, instead of the banks which have a weaker one in relation to other assets in the stock exchange.
\end{abstract}

\section{Keywords}

Random Matrix Theory, Cross-Correlations, Emerging Markets, Option Pricing, Eigenvalues Eigenvectors, Inverse Participation Ratios and Implied Volatility

\section{Introduction}

This paper investigates and compares the spectral properties of correlation matrices of price fluctuations in Nigerian and South African Stock Markets, using the Random Matrix Theory (RMT). Alternative approaches, namely factor and principal components analysis for measuring the extent of correlations could be found as presented in [1] [2] [3] [4] [5]. In this research, we use RMT to compare the empirical correlation matrix with Wishart random matrix, which models normality and departures from which connote the existence of significant market information in the observed price fluctuations [6]. 
Pafka and Kondor [6] assert that correlation matrices of financial returns play a crucial role in various aspects of modern finance including investment theory, capital allocation, and risk management. Also, [7] declare that following the introduction of RMT into the financial markets by [8] and [9], the concept has been used in the study of the statistical properties of cross-correlations in different financial markets [10]-[22]. Laurent Laloux et al. [8] opine that for financial assets, the study of the empirical correlation matrix is very relevant, since, from their finding, it is its estimation in the price movements of different assets that constitutes a significant and indispensable aspect of risk management. They declare that the probability of huge losses for a certain portfolio or option book is dominated by correlated moves of its different components and that a position which is simultaneously long in stocks and short in bonds will be risky as stocks and bonds usually move in opposite directions during crisis periods.

The interesting question that concerned investors need to answer is how volatility, which is a measure of market fluctuations, affects the dynamics of the market or vice versa. It is, therefore, expedient to explore the relationship between volatility and the coupling of stocks with one another, using correlation matrices [14]. Thus, correlations amongst the volatility of different assets are very useful, not only for portfolio selection, but also in pricing options and certain multivariate econometric models for price forecasting and volatility estimations. Engle and Figlewski [23] assert that with regards to Black-Scholes option pricing model the variance of the portfolio, $\rho$, of options exposed to Vega risk only is given by

$$
\operatorname{Var}(\rho)=\sum_{i, j, k, l} \frac{w_{i} w_{l} \Lambda_{i j} \Lambda_{l k} C_{j k}}{v_{j} v_{k} \sigma_{j} \sigma_{k}}
$$

where $w_{i}$ are the weights in the portfolio, $C_{i j}$ is the correlation matrix for the implied volatility for the underlying assets and the Vega matrix $\Lambda_{i j}$ is defined as

$$
\Lambda_{i j}=\frac{\partial p_{i}}{\partial v_{j}}
$$

with $p_{i}$ as the price of option $i, v_{j}$ is the implied volatility of asset underlying option $j$ and $\sigma_{i}$ is the standard deviation of the implied volatility $v_{i}$.

Similarly, for investors using derivatives products as a hedge on the underlying assets and for risk management, it is advisable that such investors should buy call and put options respectively for assets whose returns move in opposing directions, as may be witnessed from the calculated empirical correlation matrix. Furthermore, an accurate quantification of correlations between the returns of various stocks is practically important in quantifying risks of stock portfolios, pricing options, and forecasting. [24] note that financial correlation matrices are the key input parameters for Markowitz [25] fundamental portfolio optimization problem aimed at providing a recipe for the selection of a portfolio of assets, such that the risk associated with the investment is minimized for a given expected return. Edelman Alan [26] asserts that RMT makes it possible for a com- 
parison between the cross-correlation matrices obtained from a given number of empirical time series data for a period $T$ with an entirely random matrix $W$, otherwise known as Wishart matrix of the same size with the empirical correlation matrix, to obtain some useful information about the market(s), which is necessary for portfolio optimization and risk management. RMT predictions represent an average over all possible interactions between the constituents of the assets in a given market under consideration. The deviations from universal predictions of RMT obtained from the Wishart matrix are used in identifying the system specific, non-random properties of the system under consideration and such variations provide information about the underlying interaction of the assets. In other words, we compare the statistics of the cross-correlation coefficients of price fluctuations of stock $i$ and $j$ against a random matrix having the same symmetric properties as that of the empirical matrix. The RMT is known to distinguish the random and non-random parts of the cross-correlation matrix $C$, the non-random parts of $C$ which deviates from RMT results is known to provide information regarding the genuine collective behaviour of the stocks under consideration and indeed the entire market at large [27].

Theoretically, the comparative analyses of asset price fluctuations (hence correlation structures) between the JSE and NSM will enable us to calibrate suitable derivative models to be proposed for adoption in the NSM for portfolio optimization and risk management. This is because from the research visit embarked upon by the researchers to the Nigerian Stock Exchange (NSE) in 2014; policy makers in the NSE are taking a clue from the JSE in their proposed introduction of some pioneer derivative products and subsequently an appropriate pricing and valuation of such products in the NSM. The research into the correlations between price changes of different stocks is not only necessary for quantifying the risk in a given portfolio, but it is also of scientific interest to researchers in Economics and Financial Mathematics [28] [29]. Interestingly, interpreting the correlations between individual stocks-price changes in a given financial market can be likened to the difficulties experienced by physicists in the fifties, in interpreting the spectra of complex nuclei. Due to the enormous amounts of spectroscopic data on the energy levels that were available, which were too complicated to be analysed through model calculations, since the nature of the interactions were not known, Random Matrix Theory (RMT) was developed to take care of the Statistics of energy levels of the complex quantum systems [30] [31].

Similarly, for financial time series in a stock exchange, the nature of interactions among constituent stock are unknown, hence the need to adopt the RMT approach in exploring these interactions between individual pairs of stocks, for use in portfolio optimization and risk management. The estimation of risk and expected returns based on variance and expected returns in a given portfolio constitutes Markowitz's model [32]. In view of the fact that the statistical properties of correlations between different stocks seem to be less universal across different stock market, [17], in this paper, we first demonstrate the validity of the general predictions of RMT for the eigenvalue statistics of the correlation matrix 
and subsequently calculate the deviations, if any, of the empirical data from the Wishart matrix predictions, to identify the nature of the correlations between the individual stocks and distinguish same from those of the deviations due to randomness, in the NSM and JSE. In doing this, the period $T$ under consideration has to be relatively large enough when compared with the number of stocks or assets being considered to minimize the noise in the correlation matrix. The two sources of noise envisaged in the use of RMT in investigating the crosscorrelations of stocks in a given financial market include (a) the noise from the period length $T$ considered with respect to the number of stock and; (b) that resulting from the fact that financial time series of historical return itself is finite or bounded thereby introducing inadvertently estimation errors (noise) in the correlation matrix [6].

Szilard and Kondor [33] also observe that the effect of noise strongly depends on the ratio of stocks to the period considered, given by $r=\frac{N}{T}$, where $N$ is the number of stocks considered and $T$ the length of the available time series. They note that for the ratio $r=0.6$ and above, there will be a pronounced effect of noise on the empirical analysis as was discovered by [9] [21] [34] and that for a smaller value of $r$ ( $r=0.2$ or less); the error due to noise drops to tolerable levels. In our case for NSM $r=\frac{82}{1018}=0.08$ and that of JSE we have $r=\frac{35}{1147}=0.03$ thus both lying in the admissible region in the values of $r$. When this is done, if the eigenvalues of the empirical correlation matrix and that of the Wishart matrix lie in the same region without any significant deviations, then the stocks are said to be uncorrelated and therefore no information or deduction can be made about the nature of the market, since it is the deviations of the eigenvalues of the correlation matrix from that of the Wishart matrix that carries information about the entire market. However, if there exists at least one eigenvalue lying outside the theoretical predicted bound of the eigenvalues in the empirical correlation matrix obtained from the stock market returns, then the deviating eigenvalue(s) is(are) known to carry information about the market under consideration.

To the best of our knowledge, no such work on the comparison of stock market correlations has been carried out on African emerging markets, especially JSE and NSM which are major emerging markets in the Sub-Saharan Africa. Most of the work on such comparison has been carried out for developed markets or developed versus emerging markets, see, for instance: [10] [17] [19] [35] [36]. On the other hand, for some comparison for different stock exchanges within the same market environment, see [17].

In some sense, the JSE is gradually approaching a developed market whereas the NSM is an ideal African emerging market with no known trades on derivative products currently existing in the market, unlike the JSE where trade on derivatives has been in existence for over two decades. Option contracts were introduced in JSE in October 1992, agricultural commodity futures in 1995 and a 
fully automated trading system in May 1996, whereas in the NSM trade in derivative products are still at the formative stage, with a recently approved derivative trade on foreign exchange future under the auspices of Financial Market Derivative Quotations (FMDQ) in 2016. As the policy makers in the NSM are benchmarking themselves on the relevant trade on derivatives in JSE towards an effective take off of derivative trade in the NSM, it is pertinent to compare the asset return correlations between the two markets, to understand the similarities and differences in the statistical properties using random matrix theory.

\section{Data}

The data set consists of the daily closing prices of 82 stocks listed in the Nigerian Stock Market, NSM from $3^{\text {rd }}$ August 2009 to $26^{\text {th }}$ August 2013, giving a total of 1019 daily closing returns after removing (a) assets that were delisted, (b) those that did not trade at all or (c) are partially in business for the period under review. The stocks considered for NSM are drawn from the Agriculture, Oil and Gas, Real Estates/Construction, Consumer Goods and Services, Health care, ICT, Financial Services, Conglomerates, Industrial Goods, and Natural Resources. For the JSE, we have a total in 35 stocks selected from Top 40 shares in the Industrial Metals and Mining, Banking, Insurance, Health care, Mobil Telecommunications, Oil and Gas, Financial services, Food and Drugs, Tobacco, Forestry and Paper, Real Estate, Media, Personal Goods and Beverages, covering the period $2^{\text {nd }}$ January 2009 to $01^{\text {st }}$ August 2013 covering a similar period as that of NSM (This period was chosen for the research because that was the period when we could get the complete market information for the two stock exchanges being considered).

For the values of the daily asset prices to be continuous and to minimize the effect of thin trading, we remove the public holidays in the period under consideration and to reduce noise in the analysis, market data for the present day is assumed to be the same with the previous day for cases where there are no information on trade for any particular asset on a given date. Also, we eliminate stocks that infrequently traded within the period under review. Let $P_{i}(t)$ be the closing price on a given day $t$, for stock $i$ and define the natural logarithmic return of the index as

$$
r_{i}(t)=\ln \frac{P_{i}(t+1)}{P_{i}(t)}
$$

where $r_{i}(t)$ is the number of observations in the two stock exchanges, NSM and JSE.

\section{Theoretical Backgrounds}

\subsection{Computing Volatility}

We calculate the price changes of assets in the two markets over a time scale $\Delta t$ which is equivalent to one day and denote the price of $i$ at a time $t$ as $s_{i}(t)$ with the corresponding price change or logarithmic returns $G_{i}(t)$ over time 
scale $\Delta t$ as

$$
G_{i}(t)=\ln \left[S_{i}(t+\Delta t)\right]-\ln \left[S_{i}(t)\right]
$$

We quantify the volatility in the respective asset return as a local average of the absolute value of daily returns of indices in an appropriate time window of $\mathrm{T}$ days as

$$
v=\frac{\sum_{t=1}^{T-1}\left|G_{i}(t)\right|}{T-1}
$$

To standardize the values of obtained from Equation (4) above for all values of $i$, we normalize $G(t)_{i}$ as follows

$$
g(t)_{i}=\frac{\prec G(t)_{i}-G(t)_{i}}{\sigma_{i}}
$$

where $\sigma_{i}=\sqrt{G(t)_{i}^{2}-G(t)_{i}^{2}}$ and ... represents the average in the period studied.

From real time series data of the implied volatility surface, we can calculate the element of $N \times N$ correlation matrix $C$ as follows

$$
C_{i j}=\left\langle g_{i}(t) g_{j}(t)\right\rangle=\frac{\left\langle\left[G_{i}(t)-\left\langle G_{i}\right\rangle\right]\left[G_{j}(t)-\left\langle G_{j}\right\rangle\right]\right\rangle}{\sqrt{\left[\left\langle G_{i}^{2}\right\rangle-\left\langle G_{i}^{2}\right\rangle\right]\left[\left\langle G_{j}^{2}\right\rangle-\left\langle G_{j}^{2}\right\rangle\right]}}
$$

$C_{i j}$ lies in the range of the closed interval $-1 \leq C_{i j} \leq 1$, with $C_{i j}=0$ means there is no correlation, $C_{i j}=-1$ implies anti-correlation and $C_{i j}=1$ means perfect correlation for the empirical correlation matrix.

\subsection{Eigenvalue Spectrum of the Correlation Matrix}

As stated earlier, our aim is to extract information about the cross-correlation from the empirical correlation matrix $C$. To this end, we are going to compare the properties of $C$ with those of a random matrix; see, [9] [11] [21] [22] [37]. It can be shown from [38] that the empirical correlation matrix $\mathrm{C}$ can be expressed as

$$
C=\frac{1}{L} G G^{T}
$$

where $G$ is the normalized $N \times L$ matrix and $G^{T}$ is the transpose of $G$. This empirical matrix will be compared with a random Wishart matrix $\mathrm{R}$ given by:

$$
R=\frac{1}{L} A A^{T}
$$

to classify the information and noise in the system [22] [37], where A is an $N \times L$ matrix whose entries are independent identically distributed random variables that are normally distributed and have zero mean and unit variance.

In our bid to use the random matrix theory in portfolio optimization and (derivative) assets risk management, we should be conversant with the universal properties of random matrices. Wilcox et al. [16] assert that there are four underlying properties of random matrices which include (a) Wishart distribution 
eigenvalues from the correlation matrix, (b) Wigner surmise for eigenvalue spacing (c) the distribution of eigenvector components of the corresponding eigenvalues and finally (d) Inverse participation ratio for Eigenvector components of the resulting correlation matrix. Authors like [26] [30] [40], assert that the statistical properties of Rare known and that in particular for the limit as $N \rightarrow \infty$, and $L \rightarrow \infty$, we have that $Q=\frac{L}{N}(\geq 1)$ is fixed. The probability function $P_{r m}(\lambda)$ of eigenvalues $\lambda$ of the random correlation matrix $R$ is given by

$$
P(\lambda)=\frac{Q}{2 \pi \sigma^{2}} \frac{\sqrt{\left(\lambda_{\max }-\lambda\right)\left(\lambda-\lambda_{\min }\right)}}{\lambda}
$$

for $\lambda$ such that $\lambda_{\min } \leq \lambda \leq \lambda_{\max }$, where $\sigma^{2}$ is the variance of the elements of $A$. Here $\sigma^{2}=1$ and $\lambda_{\min }$ and $\lambda_{\max }$ satisfy

$$
\lambda_{\max / \min }=\sigma^{2}\left(1+\frac{1}{Q} \mp 2 \sqrt{1 / Q}\right)
$$

The values of lambda from Equation (10) that satisfy (11) and (12) are called the Wishart distribution of eigenvalues from the correlation matrix. These values of lambda obtained from Equation (11) as stated before determine the bounds of theoretical eigenvalue distribution. When the eigenvalues of empirical correlation matrix $\mathrm{C}$ are beyond these bounds, they are said to deviate from the random matrix bounds and are therefore supposed to carry some useful information about the market, [12].

The distribution of eigenvalue spacing was introduced as the required test for the case when there are not significant deviations of the empirical eigenvalue distribution to that of the random matrix prediction Wilcox et al. [16]. When the eigenvalues so obtained from the correlation matrix do not deviate significantly from the predictions of the RMT we apply the so-called Wigner surmise for eigenvalue spacing otherwise called Gaussian orthogonal ensemble [11] and is given by

$$
P(s)=\frac{s}{2 \pi} \exp \left(-\frac{s \pi^{2}}{4}\right),
$$

where $\left(\lambda_{i+1}-\lambda_{i}\right) / d$ and $d$ denotes the average of the differences $\lambda_{i+1}-\lambda_{i}$ as $i$ varies.

\subsection{Distribution of Eigenvector Component}

The concept that low lying eigenvalues are really random can also be verified by studying the statistical structure of the corresponding eigenvectors. The jth component of the eigenvector corresponding to each eigenvalue $\lambda_{\alpha}$ will be denoted by, $v_{\alpha, j}$ and then normalized such that $\sum_{j=1}^{N} v_{\alpha, j}^{2}=N$. Plerou et al. [9] assert that if there is no information contained in the eigenvector, $v_{\alpha, j}$, one expects that for a fixed $\alpha$, the distribution of $u=v_{\alpha, j}$ (as $j$ is varied) is a maximum entropy. This, therefore, leads to what is called Porter-Thomas distribution in the theory of random matrices written as 


$$
p(u)=\frac{1}{\sqrt{2 \pi}} \exp \left(-\frac{u^{2}}{2}\right)
$$

In line with the assumption of pure randomness and independence, the distribution of the components, $u_{a}(l)$ for $l=1,2,3, \cdots, N$ of an eigenvector $u_{a}$ of a random correlation matrix, $R$ should obey the standard normal distribution with zero mean and unit variance, [41]. The distribution so obtained from (13) above are expected to fit well the histogram of the eigenvector except for those corresponding to the highest eigenvalues which lie beyond the theoretical value of, $\lambda_{\max },[9]$.

\subsection{Inverse Participation Ratio}

Guhr, T. et al. [41] assert that to quantify the number of components that participates significantly in each eigenvector, we use inverse participation ratio (IPR). This (IPR) shows the degree of deviation of the distribution of eigenvectors from RMT results and distinguishes one eigenvector with approximately equal components with another that has a small number of huge components. For each eigenvector, $v_{a}$, [11] defined the inverse participation ratio as

$$
I_{\alpha}=\sum_{j=1}^{N}\left(v_{\alpha j}\right)^{4}
$$

where $N$ is the number of the time series (the number of implied volatility considered) and hence the number of eigenvalue components and $v_{\alpha}^{j}$ is the $j$ th component of the eigenvector, $v_{\alpha}$. There are two limiting cases of $I_{\alpha}(i)$; If an eigenvector $v_{\alpha}$ has an identical component, $v_{\alpha}(j)=\frac{1}{\sqrt{N}}$, then $I_{\alpha}=\frac{1}{N}$ and (ii) For the case when the eigenvector $v_{\alpha}$ has one element with $v_{\alpha}(j)=1$ and the remaining components zero, then $I_{\alpha}=1$. Therefore, the IPR can be illustrated as the inverse of the number of elements of an eigenvector that are different from zero that contribute significantly to the value of the eigenvector. [42] in their study of the RMT assert that the expectation of the IPR is given by

$$
\left\langle I_{\alpha}\right\rangle=N \int_{-\infty}^{\infty}\left[v_{\alpha}(j)\right]^{4} \frac{1}{\sqrt{2 \pi N}} \exp \left(-\frac{\left[v_{\alpha}(j)\right]^{2}}{2 N}\right) \mathrm{d} v_{\alpha}(j)=\frac{3}{N}
$$

since the kurtosis (extreme deviations) for a distribution of eigenvector components $s$.

\section{Empirical Result and Data Analysis}

\subsection{Eigenvalue Analysis}

We took a sample study of eighty-two $(N=82)$ stocks from the Nigerian stock exchange which gave rise to $L=1019$ daily closing prices. For the Johannesburg stock exchange, JSE we had a sample study of thirty-five $\left(N^{\prime}=35\right)$ stocks with a total of $L^{\prime}=1148$. The theoretical eigenvalue bounds in the NSM are respectively $\lambda_{-}=0.51$ and $\lambda_{+}=1.65$ as minimum and maximum values with $Q=\frac{L}{N}=12.41$. 
Further from the calculation, the market value shows that the largest eigenvalue $\lambda_{1}=4.87$ which is approximately three times larger than the predicted RMT of value (1.64). Similarly for the JSE, the theoretical eigenvalue bounds of the correlation matrix are $\lambda_{-}=0.21$ and $\lambda_{+}=2.37$ as minimum and maximum eigenvalues respectively, with $Q^{\prime}=\frac{L^{\prime}}{N^{\prime}}=32.77$. A high percentage of the eigenvalues obtained from the empirical correlation matrix of stock market price returns lie below $\lambda_{\min }\left(\lambda_{-}\right)$, just as obtained by [16] and this is attributable to the fact that many of the liquid stocks behave independently when compared with the rest of the market. The empirical market value calculations show that the largest eigenvalue $\lambda_{1}=11.86$ which is five times larger than the predicted RMT value of 2.37 above. If there were no correlations between the stocks in NSM and JSE, the eigenvalues derived from the market data would have been bounded between $\lambda_{-}=$ 0.51 and $\lambda_{+}=1.65$ for NSM and $\lambda_{-}=0.21$ and $\lambda_{+}=2.37$ for JSE respectively. In NSM 7.3\% of the eigenvalue lie outside the theoretical value and therefore contain information about the market whereas in JSE $8.57 \%$ of the total eigenvalue carry information about the entire market (see Figure 1 and Figure 2). With these significant deviations in the empirical eigenvalue distribution from the RMT predictions, the test for Wigner surmises for eigenvalue spacing are not relevant in this case.

The average $\left\langle C_{i j}\right\rangle$ of the elements of the market correlation matrix for the NSM is 0.041 , and that of the JSE is 0.168 , showing that even though the two markets are both emerging the JSE is about four times more correlated than that of the NSM. Thus, this shows that the Johannesburg market is much more emerging than the Nigerian market, [10]. It, therefore, means that since many assets in JSE are more correlated than that of the NSM, perhaps different macroeconomic forces are driving the two markets, [19]. It is also worthy of mention that the empirical correlation matrices obtained from the two markets are positive definite since all the eigenvalues obtained are all positive.

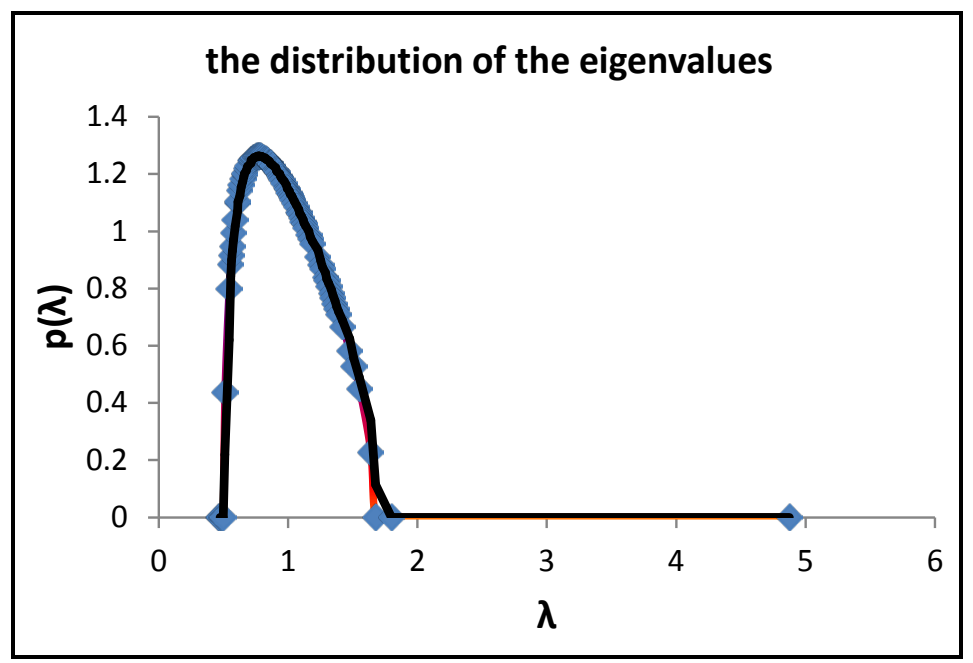

Figure 1. Theoretical (Marcenko-Pastur) empirical eigenvalues for NSM (source: Nigerian Stock Market price return 2009-2013). 


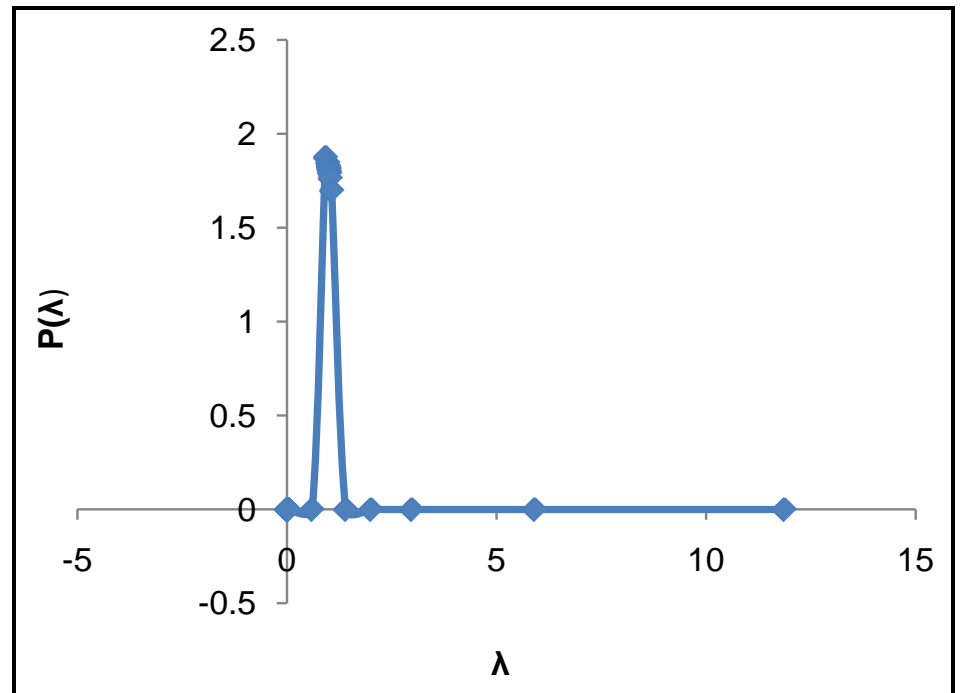

Figure 2. Theoretical (Marcenko-Pastur) empirical eigenvalues for JSE (source: Johannesburg Stock Exchange price return 2009-2013).

The comparable informative indices (7.3\% and $8.6 \%)$ for NSM and JSE, respectively, suggest a similarity between the market microstructures in the system.

Figure 3 above represent the distribution of eigenvectors for the various eigenvalues in the empirical correlation matrix of the NSM. The eigenvector labelled U1 and U82 represents an eigenvector for deviating eigenvalue in the theoretical (hypothetical) region whereas the other 4 diagrams are the eigenvector components of the eigenvalue within the regions predicted from the Random Matrix Theory.

The overwhelming non-informativeness of the remaining $92.7 \%$ and $91.4 \%$ of the overall markets, further suggests typical random behaviour of the two markets. Typically, the distribution of the first three eigenvectors indicates the key features (mean, standard deviation and kurtosis) of a market. A look at these first three distributions for the NSM shows compared to the normal distribution, they are skewed and leptokurtic in mean and standard deviations, but fairly symmetric in kurtosis. The JSE versions portray similar non-symmetric behaviours, but fairly symmetric in kurtosis. The NSM distributions would seem to follow a beta-gamma family of distribution while the JSE ones are mostly negatively skewed, as opposed to the first two NSM distributions which are positively skewed. In general, higher-order distributions are examined for a more detailed understanding of market-dynamics, for example, market microstructure.

These distributions present the same profiles as the first three distributions in the two markets, which suggest persistence of market features and the driving economic forces. Given the fact the distributions reveal the presence of market information outside the noisy RMT range; the results suggest potential market inefficiency and ability to make money from the markets. We cannot, however, say more that this regarding the stylised fats and market features, without a detailed examination of the key financial economics features typically explored in 
empirical finance, namely market efficiency, volatility, bubbles, anomalies, valuations and predictability.

Figure 4 shows the eigenvector distribution for some eigenvalues within and outside the theoretical region of the Random Matrix Theory. The last diagrams
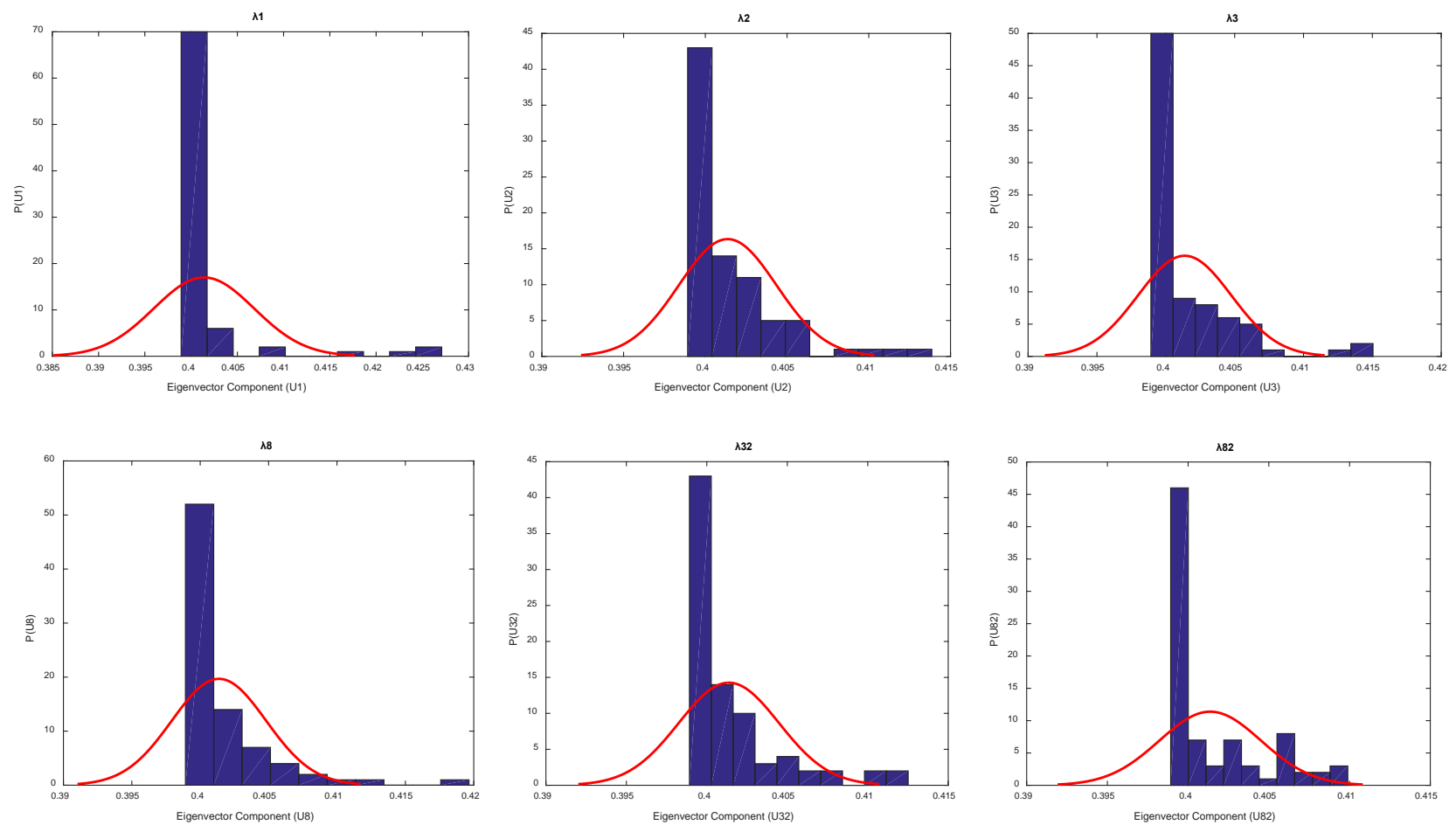

Figure 3. Distribution of eigenvector components of stocks in NSM. Source [43].
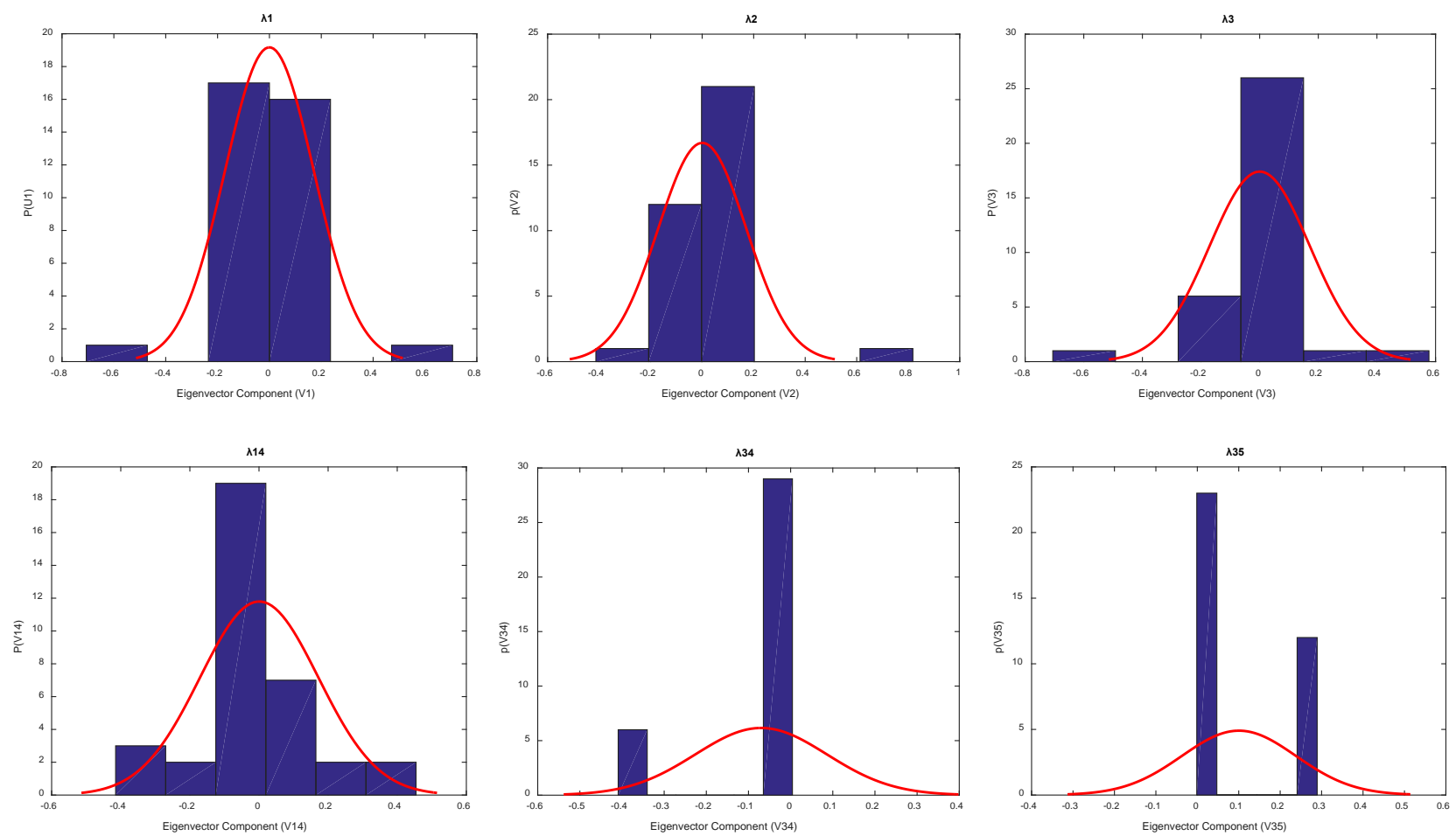

Figure 4. Distribution of eigenvector components of stocks in JSE. Source: [44]. 
V34 and V35 represent the eigenvectors corresponding to an eigenvalue outside the region predicted by RMT which contain the information about the market. The other eigenvectors correspond to the eigenvalues due to noise as they lie in the region predicted by RMT.

The key interest in this paper is to assess how similar the NSM and JSE are, to facilitate future modelling of as yet non-existent derivative prices in the NSM using available information on existing derivative prices in the JSE. For this, a comparative look at the two sets of eigenvector distributions suggest a flipping over or reverse dynamics in the JSE in comparison with the NSM. For example, the $\mathrm{U} 2$ and $\mathrm{U}_{3}(\mathrm{NSM})$ versus $\mathrm{V} 2$ and $\mathrm{V}_{3}(\mathrm{JSE})$ eigenvalue distributions are mirror reflections of each other. The practical implication of this reveals that different market forces seem to drive the NSM and JSE. This result is intuitively meaningful because the NSM is an oil-dependent and erratic in its price dynamics and market microstructure unlike the JSE which is mining dependent, and is therefore relatively stable in nature. Consequently, attempts to model, say, non-existent derivative prices in Nigeria using existing prices in the JSE have to be taken cautiously. That said, the flipping-over features suggest that including NSM and JSE stocks in an African Emerging Markets portfolio would achieve reasonable portfolio diversification and corresponding Markowitz-style mean-variance portfolio optimization. These insights reveal the power of statistical physics tools such as RMT in peering through complex market dynamics which may not manifest with traditional mathematical finance techniques.

\subsection{Inverse Participation Ratios (IPRs)}

The inverse participation ratio (IPR) is the multiplicative inverse of the number of eigenvector components that contribute significantly to the eigenmode, [11]. For the largest eigenvalue nine deviating from the RMT bounds, almost all the stocks contribute to the corresponding eigenvector thereby justifying treating this eigenvector as the market factor. The eigenvector corresponding to other deviating eigenvalues also exhibits that their corresponding stocks contribute slightly to the overall market features in the two exchanges, NSM and JSE.

The average IPR value is around $3 / 82$ for NSM \& 1/35 for JSE respectively larger than would be expected $1 / \mathrm{N}=1 / 82=0.01$ for $\mathrm{NSM} \& 1 / 35=0.03$ for JSE, if all components contributed to each eigenvector, [37]. The remaining eigenvectors appear to be random with some deviations from the predicted value of $3 / \mathrm{N}=0.04$ and 0.09 respectively for NSM and JSE possibly as a result of the existence of fat tails and high kurtosis of the return distributions.

The lower end of JSE and the higher end of the eigenvalues for both exchanges (NSM and JSE) show deviations suggesting the existence of localized modes. It is noticeable from Figure 5 and Figure 6 that these deviations are fewer in number for JSE than that of the NSM, which implies that distinct groups whose members are mutually correlated in their price movements are witnessed in both markets although they are more noticeable in JSE. 


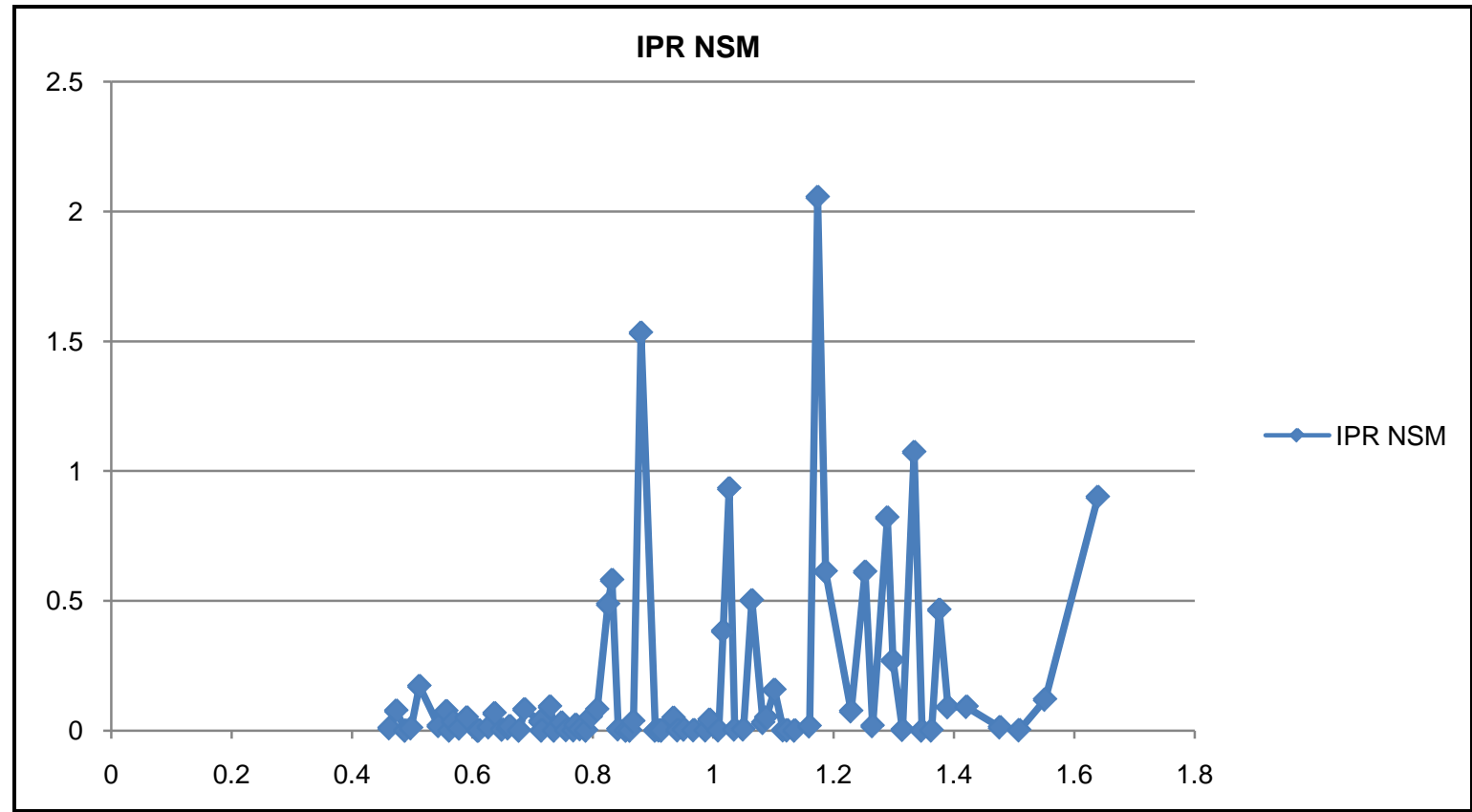

Figure 5. Inverse participation ratio and their ranks for NSM. Source: [43].

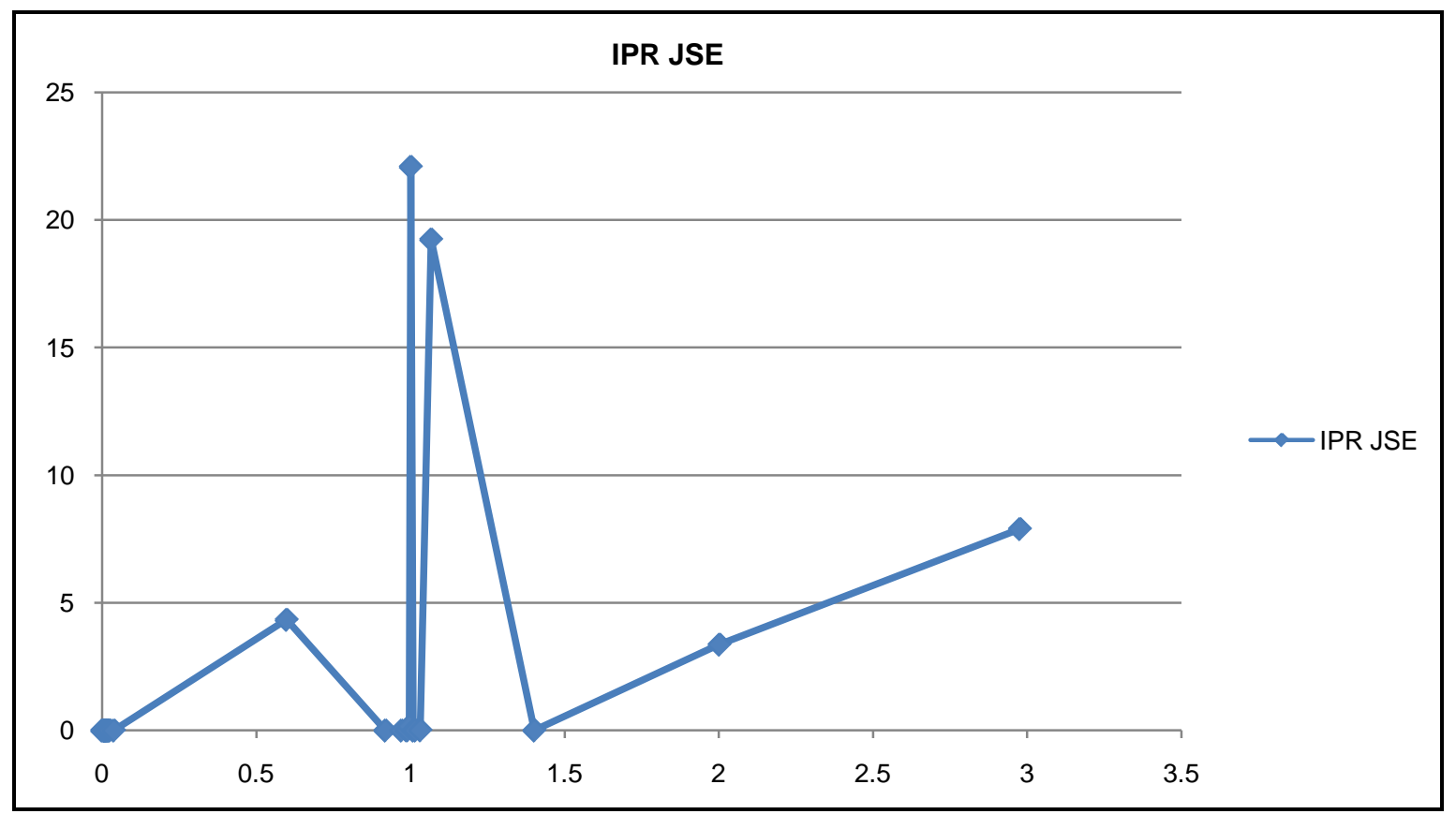

Figure 6. Inverse participation ratio and their ranks for JSE. Source: [44].

\subsection{Contributions to Knowledge}

This paper stems from a doctoral research which aims to model yet non-existing derivative prices in the NSM, using existing prices in the JSE. The underpinning heuristics (not developed in detail in this paper) is to backtrack from measures of similarity or dissimilarity between the stylized facts and other empirical correlates of the two market dynamics, one of which is the random matrix correlation structures. The paper, therefore, is novel in foregrounding the modeling of 
non-existing financial derivatives for the first time known to the authors.

\subsection{Limitations of the Study}

It would have been preferable to use up to date data (2009-2016) for the two markets to accommodate the recent impact of oil price fluctuation on the market dynamics. This was not possible since for the NSM available data from the Nigerian Stock Exchange when this research was being carried out range from 2009-2013. The authors therefore, used this range that was available for the analysis. Strictly speaking from the point of using the results in derivative pricing, this limitation is not severe as one can forecast parts of the data that are not available or simulate alternative impact scenarios for the revealed price paths of crude oil between 2013 and 2016, for example.

\section{Conclusion and Hints on Future Work}

The analysis of the correlation and structure of stock market returns for the two most dominant markets in the Sub-Saharan Africa, NSM and JSE, was carried out in this paper using RMT. Marcenko-Pastur eigenvalue distribution predicted that the theoretical eigenvalues should be in the range of 0.52 and 1.65 for NSM and 0.21 and 2.37 for JSE respectively. While for NSM it was observed that 6 out of 82 stocks considered that have their corresponding eigenvalues lie outside this theoretical bound of eigenvalues, in JSE 3 out of the 35 stocks has their eigenvalues outside the predicted eigenvalue bounds. Therefore, $89 \%$ of the information from the return distributions is purely random thereby leaving us with the alternative hypothesis of the RMT which states that the information on the market lies on the deviating eigenvalues which imply then that for NSM the true market characteristic lies with only $11 \%$ of the assets examined. Similarly, for JSE, only $9 \%$ of the stocks considered have information about the market which can be used in constructing portfolios with better stable returns and optimal risk management. As stated earlier, these correlation matrices contain some relevant information for option pricing and hedging [45].

We noted earlier in the literature review that random matrix theory could be very useful in options trading, hedging and in the management of risks associated with a portfolio of investment. In this regard, we intend to use the RMT results in this paper to construct suitable investment portfolios from overall market and sector-based results, for given weights and implied volatilities of the stocks in the respective portfolios under consideration. As Nigeria is yet to commence trade on derivative products, we will carry out heuristic analyses of the option price data for NSM, and execute same for JSE using obtained data from the Johannesburg Stock Exchange, to adjudicate the relative performances of different derivative pricing models in the two markets.

\section{References}

[1] Gentle, J. (1998) Numerical Linear Algebra with Applications in Statistics. Statistics and Computing, Springer, Berlin. https://doi.org/10.1007/978-1-4612-0623-1 
[2] Gentle, J. (1998) Singular Value Factorization. Springer, Berlin.

[3] Morrison, D.F. (1990) Multivariate Statistical Methods. 3rd Edition, McGraw-Hill, Inc., New York, 1-85.

[4] Jackson, E. (2003) A User's Guide to Principal Components. John-Wiley and Sons Inc., New York, 1-65.

[5] Cont, R. and Fonseca, J. (2002) Dynamics of Implied Volatility Surfaces. Quantitative Finance, 2, 45-60. https://doi.org/10.1088/1469-7688/2/1/304

[6] Pafka, S. and Kondor, I. (2004) Estimated Correlation Matrices and Portfolio Optimization. Physica A, 343, 623-634.

[7] Wang, G., Xie, C., Chen, S., Yang, J. and Yang, M. (2013) Random Matrix Theory Analysis of Cross-Correlations in the US Stock Market: Evidence from Pearson's Correlation Coefficient and Detrended Cross-Correlation Coefficient. Physica A, 392, 3715-3730.

[8] Laloux, L., Cizeau, P., Bouchaud, J. and Potters, M. (1999) Noise Dressing of Financial Correlation Matrices. Physical Review Letters, 83, 1467-1470. https://doi.org/10.1103/PhysRevLett.83.1467

[9] Plerou, V., Gopikrishnan, P., Rosenow, B., Amaral, L.A.N. and Stanley, H.E. (1999) Universal and Non-Universal Properties of Cross-Correlations in Financial Time Series. Physical Review Letters, 83, 1471-1474. https://doi.org/10.1103/PhysRevLett.83.1471

[10] Shen, J. and Zheng, B. (2009) Cross-Correlation in Financial Dynamics. A Letters Journal Exploring the Frontiers of Physics, 86, 48005. https://doi.org/10.1209/0295-5075/86/48005

[11] Plerou, V., Gopikrishnan, P., Rosenow, B., Amaral, L.A.N., Guhr, T. and Stanley, H.E. (2002) Random Matrix Approach to Cross-Correlations in Financial Data. Physical Review E, 65, Article ID: 066126. https://doi.org/10.1103/physreve.65.066126

[12] Cukur, S., Eryigit, M. and Eryigit R. (2007) Cross-Correlation in an Emerging Market Financial Data. Physica A, 376, 555-564.

[13] Junior, L. and Franca, I. (2012) Correlation of Financial Markets in Times of Crisis. Physica A, 391, 187-208.

[14] Kulkarni, V. and Deo, N. (2007) Correlation and Volatility in an Indian Stock Market: A Random Matrix Approach. EuroPhysics Journal B, 60, 101-109.

[15] Sharma, C. and Banerjee, K. (2015) A Study of Correlation in the Stock Market. Physica A: Statistical Mechanics and Its Applications, 321-330.

[16] Wilcox, D. and Gebbie, T. (2007) An Analysis of Cross-Correlations in an Emerging Market. Physica A, 375, 584-598.

[17] Kumar, R. and Sinha, S. (2007) Collective Behaviour of Stock Price Movements in an Emerging Market. Physical Review E, 76, Article ID: 046116.

[18] Kim, M., Lee, S., Hwang, D., Kim, S. and Ko, I. (2010) Dynamics of Implied Volatility Surfaces from Random Matrix Theory. Physica A, 389, 2762-2769.

[19] Fenn, D.J., Porter, M., Williams, S., McDonald, M., Johnson, N. and Jones, N. (2011) Temporal Evolution of Financial Market Correlations. Physical Review E, 84, Article ID: 026109. https://doi.org/10.1103/physreve.84.026109

[20] Nobi, A., Maeng, S., Gyun, G. and Lee, J. (2013) Random Matrix Theory and CrossCorrelations in Global Financial Indices and Local Stock Market Indices. Journal of the Korean Physical Society, 62, 569-574. https://doi.org/10.3938/jkps.62.569

[21] Laloux, L., Cizeau, P. and Potters, M.J. (2000) Bouchaud, Random Matrix Theory 
and Financial Correlations. International Journal of Theoretical and Applied Finance, 3, 1-7. https://doi.org/10.1142/S0219024900000255

[22] Gopikrishnan, P., Rosenow, B., Plerou, V. and Stanley, E. (2001) Quantifying and Interpreting Collective Behaviour in Financial Markets. Physical Review E, 64, Article ID: 035106.

[23] Engle, R. and Figlewski, S. (2014) Modeling the Dynamics of Correlation among Implied Volatilities. REV Finance Group, Inc., Arcadia, 1-28.

[24] Plerou, V., Gopikrishnan, P., Rosenow, B., Amaral, L.A.N. and Stanley, H.E. (2000) A Random Matrix Theory Approach to Financial Cross-Correlations. Physica A, 287, 374-382.

[25] Markowitz, H. (1952) Portfolio Selection. Journal of Finance, 2, 77-91. https://doi.org/10.1111/j.1540-6261.1952.tb01525.x

[26] Edelman, A. (1988) Eigen Values and Condition Numbers of Random Matrices. SIAM Journal on Matrix Analysis and Applications, 9, 543-560. https://doi.org/10.1137/0609045

[27] Plerou, V., Gopikrishnan, P., Rosenow, B., Amaral, L.A.N. and Stanley, H.E. (2001) Collective Behaviour of Stock Price Movements: A Random Matrix Theory Approach. Physica A, 299, 175-180.

[28] Gee-Rae, K. and Markowitz, H.M. (1989) Investment Rules, Margin and Market Volatility. The Journal of Portfolio Management, 6, 45-52.

[29] Palmer, R.G., Arthur, W.B., Holland, J.H. and LeBaron, B. (1994) Artificial Economic Life: A Simple Model of a Stock Market. Physica D, 75, 264-274.

[30] Kondor, I. and Kertesz, J. (1999) EconoPhysics: An Emerging Science. Kluwer, Dordrecht.

[31] Chatterjee, A. and Chakrabarti, B.K. (2006) Econophysics of Stock and Other Markets. Springer, Berlin. https://doi.org/10.1007/978-88-470-0502-0

[32] Markowitz, H. (1959) Portfolio Selection: Efficient Diversification of Investment. John Wiley and Sons, New York.

[33] Pafka, S. and Kondor, I. (2003) Noisy Covariance Matrices and Portfolio Optimization 2. Physica $A, 319,487-494$.

[34] Galluccio, S., Bouchaud, J. and Potters, M. (1998) Rational Decisions, Random Matrices and Spin Glasses. Physica A, 259, 449-456.

[35] Wang, D., Podobrink, B., Horvatic, D. and Stanley, H.E. (2011) Quantifying and Modeling Long-Range Cross-Correlations in Multiple Time Series with Applications to World Stock Indices. Physical Review E, 83, Article ID: 046121. https://doi.org/10.1103/PhysRevE.83.046121

[36] Sensoy, A., Yuksel, S. and Erturk, M. (2013) Analysis of Cross-Correlations between Financial Markets after the 2008 Crisis. Physica A, 392, 5027-5045.

[37] Colon, T., Ruskin, H.J. and Crane, M. (2007) Random Matrix Theory and Fund of Funds Portfolio Optimization. Physica A, 382, 565-576.

[38] Sharifi, S., Crane, M., Shamaie, A. and Ruskin, H. (2004) Random Matrix Theory for Portfolio Optimization: A Stability Approach. Physica A, 335, 629-643.

[39] Sengupta, A.M. and Mitra, P.P. (1999) Distributions of Singular Values for Some Random Matrices. Physical Review E, 60, 1-4. https://doi.org/10.1103/PhysRevE.60.3389

[40] Bai, Z.D. (1999) Methodologies in Spectral Analysis of Large Dimensional Random Matrices: A Review. Statistica Sinica, 9, 611-677. 
[41] Guhr, T., Muller, A. and Weidenmuller, H. (1998) Random Matrix Theories in Quantum Physics: Common Concepts. Physics Reports, 299, 189-425.

[42] Utsugi, A., Ino, K. and Oshikawa, M. (2004) Random Matrix Theory Analysis of Cross-Correlations in Financial Markets. Physical Review E, 70, Article ID: 026110. https://doi.org/10.1103/physreve.70.026110

[43] Closing Prices of Some Stocks from the Nigerian Stock Market Official Data Obtained through the Nigerian Stock Exchange.

[44] Closing Prices of Some Stocks Selected from the 40 Most Actively Traded Stocks on the Johannesburg Stock Exchange, South Africa, Obtained from the Johannesburg Stock Exchange.

[45] Hull, J. (1997) Options, Futures and Other Derivative Securities. Prince Hall, Englewood Cliffs.

Submit or recommend next manuscript to SCIRP and we will provide best service for you:

Accepting pre-submission inquiries through Email, Facebook, LinkedIn, Twitter, etc. A wide selection of journals (inclusive of 9 subjects, more than 200 journals)

Providing 24-hour high-quality service

User-friendly online submission system

Fair and swift peer-review system

Efficient typesetting and proofreading procedure

Display of the result of downloads and visits, as well as the number of cited articles

Maximum dissemination of your research work

Submit your manuscript at: http://papersubmission.scirp.org/

Or contact jmf@scirp.org 